\title{
Phenotype-phenotype and genotype-phenotype correlations in patients with idiopathic superior oblique muscle palsy
}

\author{
Sayuri Imai Ohkubo, Toshihiko Matsuo, Kayoko Hasebe, Yoshie Hirai Shira, Emi Itoshima \\ and Hiroshi Ohtsuki
}

Idiopathic superior oblique muscle palsy presents, as quantitative phenotypes, vertical deviation and cyclodeviation in eye alignment on clinical testing, and superior oblique muscle hypoplasia on imaging. We determined $A R I X$ and PHOX2B polymorphisms as genotypes, and analyzed phenotype-phenotype and genotype-phenotype correlations in 37 patients with idiopathic superior oblique muscle palsy. Vertical deviations were measured at upright position of the head and head tilt for $30^{\circ}$ to either side, and angles of objective excyclodeviations were determined by image analysis on fundus photographs. Crosssectional areas of the superior oblique muscle near the eye globe-optic nerve junction were measured by image analysis on coronal sections of magnetic resonance imaging to calculate the paretic-side/normal-side ratios. Among the phenotypes, the increase in vertical deviations elicited by head tilt to the paretic side, the decrease in vertical deviations elicited by head tilt to the normal side and the difference of angles of objective excyclodeviations between the paretic side and normal side were significantly correlated inversely with the paretic-side/normal-side ratios of the cross-sectional areas of the muscle $(r=-0.43$ with $P=0.0084, r=-0.34$ with $P=0.038$, and $r=-0.43$ with $P=0.009$, respectively, $n=37$, Pearson's correlation test). Fifteen patients with $A R I X$ and/or $P H O X 2 B$ polymorphisms had significantly greater paretic-side/normal-side ratios of the muscle compared with 20 patients without the polymorphisms $(P=0.017, n=35$, Mann-Whitney $U$-test). The patients with $A R I X$ and/or PHOX2B polymorphisms had less hypoplastic superior oblique muscles.

Journal of Human Genetics (2012) 57, 122-129; doi:10.1038/jhg.2011.138; published online 15 December 2011

Keywords: ARIX (PHOX2A); congenital or idiopathic superior oblique muscle palsy; cyclotorsion (cyclodeviation); genotype-phenotype; magnetic resonance imaging; muscle hypoplasia; $P H O X 2 B$; quantitative phenotype

\section{INTRODUCTION}

Idiopathic or congenital superior oblique muscle palsy is a common form of incomitant strabismus. Its etiology remains unknown but genetic contribution is suggested based on the familial occurrence $e^{1-5}$ and structural abnormalities such as muscle aplasia and hypoplasia. ${ }^{6-14}$ The clinical features of idiopathic superior oblique muscle palsy are hypertropia (upward deviation of the eye), underaction of the superior oblique muscle and overaction of the unopposed antagonist inferior oblique muscle, abnormal head posture such as head tilt toward the non-paretic side, the Bielschowsky head tilt phenomenon, the presence of large vertical fusional amplitudes and, rarely, amblyopia in the non-dominant eye.

Genetic understanding of congenital cranial dysinnervation disorders, including congenital fibrosis of the extraocular muscles (CFEOM) and idiopathic superior oblique muscle palsy, has advanced in recent years. ${ }^{15}$ KIF21A gene mutations have been identified in patients with CFEOM type 1, which shows an autosomal dominant trait and is associated with the absence of the superior branch of the oculomotor nerve. ${ }^{16}$ ARIX (PHOX2A) gene mutations have been found in patients with CFEOM type 2, which shows an autosomal recessive trait and is proposed to result from aberrant development of the oculomotor and trochlear nerve, and nuclei in the brainstem. ${ }^{17} \mathrm{On}$ the basis of working hypothesis that idiopathic superior oblique muscle palsy might be a clinically milder variant of CFEOM type 2, we previously analyzed $A R I X$ and $P H O X 2 B$ gene polymorphisms in patients with idiopathic superior oblique muscle palsy. ${ }^{5,18,19}$ The PHOX2B gene is a homolog of the ARIX gene, $100 \%$ identical to $A R I X$ in the homeodomain region and $75 \%$ identical in the brachyury-like domain. ${ }^{20}$ We indeed found that $A R I X$ and $P H O X 2 B$ gene polymorphisms might be genetic risk factors for the development of idiopathic superior oblique muscle palsy by comparing the patients with normal subjects. ${ }^{5,18,19}$ The allele frequency of ARIX $153 \mathrm{G}$ to A in the $5^{\prime}$-untranslated region of the exon 1 (rs140850664) was higher in patients with idiopathic superior oblique muscle palsy $(8 / 31=0.26)$ 
compared with normal subjects $(4 / 54=0.07)^{18,19}$ while $P H O X 2 B$ $1121 \mathrm{~A}$ to $\mathrm{C}$ in the exon 3 (rs17884724) was found only in patients with idiopathic superior oblique muscle palsy, but not in the normal subjects. ${ }^{19}$

In this study, we chose, as quantitative phenotypes, namely, quantitative traits, vertical deviation and cyclodeviation in eye alignment on clinical testing as well as the extent of the superior oblique muscle hypoplasia on imaging, and analyzed ARIX and PHOX2B polymorphisms as genotypes in patients with idiopathic superior oblique muscle palsy. We aimed to find reliable quantitative phenotypes by phenotype-phenotype correlation analysis and also to get insight into the role of ARIX and PHOX2B polymorphisms in these quantitative phenotypes by genotype-phenotype correlation analysis of the disease.

\section{MATERIALS AND METHODS}

\section{Patients}

The study was approved by the Institutional Review Board at Okayama University Medical School, and written consent was obtained from each patient or a parent when the patient was below the age 15. All of the procedures conformed to the Declaration of Helsinki. This study involved 37 patients who were diagnosed with idiopathic superior oblique muscle palsy on a unilateral side at Okayama University Hospital from 1996 to 2008, and underwent orbital magnetic resonance imaging. The patients were 21 males and 16 females, with the age at the initial visit ranging from 3 to 79 years (mean and standard deviation, $32 \pm 24$ years). The palsy was on the right side in 22 patients while on the left side in 15 patients. The dominant (fixating) eyes were on the paretic side in 22 patients while on the normal side in 15 patients. Orbital magnetic resonance imaging was performed preoperatively in all patients to evaluate the status of the superior oblique muscle. ${ }^{14}$ The patients were instructed to close the eyes during the imaging. Genomic DNA from these 37 patients was used for the study.

Of these 37 patients, 35 patients underwent surgery and the remaining 2 patients were followed without surgery. The superior oblique muscle aplasia was found on the right side in one male patient and on the left side in another male patient. In the same 13-year period, 269 consecutive patients in total were diagnosed as congenital or idiopathic superior oblique muscle palsy on a unilateral side (266 patients) or on both the sides (three patients) at Okayama University Hospital. Of the 269 patients with the palsy, 168 patients were excluded from the study because their genomic DNA was not available. Of the remaining 101 patients with the genomic DNA available, 64 patients were excluded from this study because (1) magnetic resonance imaging was not done in 26 patients while magnetic resonance imaging was done at other hospitals and the films were not available in 21 patients, (2) objective excyclodeviation was not measured because of young ages or for unknown reasons in 5 patients, and (3) the measurement of vertical deviations was unreliable because of masked or apparent bilateral superior oblique muscle palsy in 3 patients, dissociated vertical deviation in 6 patients, and past history of extraocular muscle surgeries in 3 patients.

\section{Standardized clinical examinations}

All patients were questioned about the age at onset, a history of previous head trauma and family history of strabismus, and patients with acquired superior oblique muscle palsy such as traumatic, ischemic and vascular accident-related palsy were excluded. In older patients, their photographs at earlier ages were obtained to check abnormal head postures to confirm the presence of the palsy from childhood. It was, in general, difficult to determine the exact age at onset because idiopathic superior oblique muscle palsy was diagnosed based on the presence of strabismus or abnormal head posture in the childhood, noted by family members.

Clinical examinations included visual acuity, slit-lamp biomicroscopic and funduscopic examinations, tonometry, inspection of head posture, deviation measurement at 5 and $0.3 \mathrm{~m}$ by alternate prism and cover test in nine diagnostic positions of the gaze, version and Bielschowsky head-tilt test, and stereoacuity testing at $0.3 \mathrm{~m}$ by the TNO Test for Stereoscopic Vision (Clement Clarke International, Ltd., Harlow, UK).

\section{Objective vertical deviations as a phenotype}

Vertical deviations, measured by alternate prism and cover tests to a light target placed at $5 \mathrm{~m}$, were used: vertical deviations at upright position of the head and at head tilt to the paretic side or normal side for $30^{\circ}$. Vertical deviations at upright position, the difference between vertical deviations at head tilt to the paretic side and at upright position, and the difference between vertical deviations at upright position and at head tilt to the normal side were used for correlation analyses.

\section{Objective excyclodeviation as a phenotype}

The objective excyclodeviation was measured by the scanning laser ophthalmoscope (Rodenstock, Munich, Germany). ${ }^{21,22}$ The patient's head was placed on the chin rest, and care was taken to eliminate obvious head tilt. The heads of younger patients were kept upright by an assistant. With both eyes open, the fundus of each eye was recorded while the patient was instructed to fixate on the ophthalmoscope's internal fixation target.

Print images of the fundus photographs were scanned and stored as Bitmap image file format. Using Scion Image for Windows software (Scion Corporation, Frederick, MD, USA), the rim of the optic disc was traced manually with a mouse on each fundus photograph and its gravitational center was determined. A horizontal line was drawn at the center of the optic disc, and a second line was placed between the center of the optic disc and the foveal center as marked by a cross on the photograph. An angle formed between the first horizontal line and the second line was measured to obtain the angle of excyclodeviation (Figure 1). ${ }^{23}$

Statistical analyses were done for angles of excyclodeviation on the paretic side and also for the sum of angles of excyclodeviation on both the sides and the difference obtained by reducing the angle on the normal side from that on the paretic side of each patient.

\section{Paretic-side/normal-side ratios of cross-sectional areas of superior oblique muscle as a phenotype}

A coronal section slice at the nearest location, posterior to the eye globe and optic nerve junction, was chosen from T1-weighted orbital magnetic resonance images. These magnetic resonance images were not quasi-coronal (namely, perpendicular to the orbital long axis), were taken by different machines with different slice thickness and were printed on films with varying magnifications. The selected slices of the magnetic resonance imaging films placed on a show case were captured by a digital camera at as highest magnification as possible with good focus, and captured images were transferred to a computer. Using

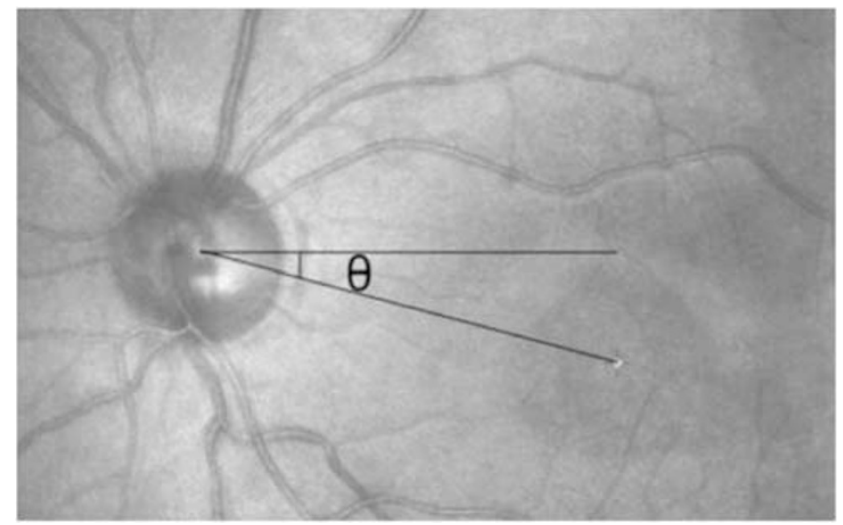

Figure 1 Measurement of angles of objective excyclodeviation on the fundus photograph obtained by scanning laser ophthalmoscope. An angle formed between horizontal line placed at the gravitational center of the optic disc and the line connecting between the foveal center and the center of the optic disc is measured to obtain the angle $(\theta$, in degrees) of excyclodeviation. 
Scion Image for Windows software, cross-sections of the superior oblique muscle were encircled manually with a mouse and the number of square pixels in the encircled area was measured to obtain the area of the cross-section. The measurements were repeated five times and a mean was calculated to get a representative value for the cross-sectional area of the muscle. ${ }^{14}$ In each patient with either right side or left side superior oblique muscle palsy, the value on the paretic side was divided by the value on the contralateral normal side to obtain the paretic-side/normal-side ratios.

\section{$A R I X$ and $P H O X 2 B$ polymorphisms as genotypes}

Peripheral leukocytes were isolated from $10 \mathrm{ml}$ blood by gradient centrifugation, and the genomic DNA was purified by phenol/chloroform extraction and ethanol precipitation. PCR amplification for the ARIX and PHOX2B genes was performed as described previously. ${ }^{5,18,19}$ Briefly, PCR products were purified and used as a template for direct sequencing with the ABI 310 Genetic Analyzer (Perkin-Elmer, Foster, CA, USA), using the BigDye Terminator Cycle Sequencing Kit (Perkin-Elmer). Both the strands were sequenced for each DNA fragment. The DNA sequences were aligned with the published human ARIX sequence (GenBank Accession Number: NM_005169), and PHOX2B sequence (GenBank Accession Number: NM_003924).

The genotype-phenotype correlation was analyzed in 35 patients because the sequences were not obtained in 2 of the 37 patients, 1 male and 1 female with paretic-side/normal-side ratios of cross-sectional areas of the superior oblique muscle as 0.9 and 0.19 , respectively.

\section{RESULTS}

\section{Phenotype-phenotype correlation}

Quantitative phenotypes are summarized in Table 1. In 37 patients with idiopathic superior oblique muscle palsy on a unilateral side, paretic-side/normal-side ratios of cross-sectional areas of the superior oblique muscle were significantly correlated inversely with the difference between vertical deviations at head tilt to the paretic side and at upright head position $(r=-0.43, P=0.0084$, Pearson's correlation test, Figure 2, Table 1), and also with the difference between vertical deviations at the upright head position and at head tilt to the normal side $(r=-0.34, P=0.038$, Figure 3 , Table 1$)$. In contrast, there was no significant correlation between paretic-side/ normal-side ratios of cross-sectional areas of the superior oblique muscle and vertical deviations at upright head position $(r=-0.24$, $P=0.15$, Table 1$)$.

No significant correlation was noted between paretic-side/normalside ratios of cross-sectional areas of the superior oblique muscle and angles of objective excyclodeviation on the paretic side $(r=-0.29$, $P=0.08$, Table 1 ). The sum of angles of objective excyclodeviation on both the sides also had no significant correlation with the muscle cross-sectional area ratios $(r=0.08, P=0.66$, Table 1$)$. In contrast, the difference of angles of objective excyclodeviation between the paretic side and the normal side (paretic-side value minus normal-side value) was significantly correlated inversely with the muscle cross-sectional area ratios $(r=-0.43, P=0.009$, Figure 4 , Table 1$)$.

\section{Genotype-phenotype correlation}

In 35 patients with idiopathic superior oblique muscle palsy with the genotypes obtained (Table 2), 15 patients with $A R I X$ and/or $P H O X 2 B$ polymorphisms had significantly larger paretic-side/normal-side ratios of cross-sectional areas of the superior oblique muscle, and significantly smaller difference between vertical deviations at upright position and at head tilt to the normal side, compared with 20 patients with no polymorphism $(P=0.017$ and $P=0.028$, respectively, MannWhitney $U$-test, Figure 5, Table 3).

In the separate analyses for ARIX or $P H O X 2 B$ polymorphisms (Table 3), 5 patients with the ARIX polymorphism had significantly smaller difference between vertical deviations at upright position and at head tilt to the normal side $(P=0.034)$ compared with 30 patients with no polymorphism. In addition, 12 patients with the $P H O X 2 B$ polymorphisms showed significantly larger sum of angles of objective

Table 1 Vertical deviations, excyclodeviations, the extent of muscle hypoplasia, and eye movement abnormalities in 37 patients with idiopathic superior oblique muscle palsy

\begin{tabular}{|c|c|c|c|}
\hline & Mean \pm s.d. & Range (median) & $\begin{array}{c}\text { Correlation }(r) \text { with muscle } \\
\text { cross-sectional area } \\
\text { ratios }(\mathrm{P} \text {-value })^{\mathrm{a}}\end{array}$ \\
\hline Vertical deviations at upright position of head (prism diopter) & $20.6 \pm 8.6$ & $4-37.5(18)$ & $-0.24(0.15)$ \\
\hline $\begin{array}{l}\text { Difference between vertical deviations at head tilt to the paretic side and at upright position } \\
\text { (prism diopter) }\end{array}$ & $8.0 \pm 8.0$ & -3 to $28(7)$ & $-0.43(0.0084)$ \\
\hline $\begin{array}{l}\text { Difference between vertical deviations at upright position and at head tilt to the normal side } \\
\text { (prism diopter) }\end{array}$ & $10.8 \pm 8.9$ & 0 to $37.5(9)$ & $-0.34(0.038)$ \\
\hline Angle of objective excyclodeviation on paretic side (degree) & $9.3 \pm 5.2$ & -5.3 to $19.0(9.4)$ & $-0.29(0.08)$ \\
\hline Sum of angles of excyclodeviation on both the sides (degree) & $19.4 \pm 5.7$ & 8.9 to $30.8(19.3)$ & $0.08(0.66)$ \\
\hline $\begin{array}{l}\text { Difference of angles of excyclodeviation between paretic side and normal side } \\
\text { (paretic-side value minus normal-side value) (degree) }\end{array}$ & $-0.7 \pm 8.1$ & -20.4 to $15.8(-0.59)$ & $-0.43(0.009)$ \\
\hline Paretic-side/normal-side ratios of cross-sectional areas of the superior oblique muscle & $0.66 \pm 0.30$ & $0-1.02(0.67)$ & \\
\hline Eye movement abnormalities & Number of patients & & \\
\hline Overaction of inferior oblique muscle on paretic side & 23 & & \\
\hline Underaction of superior oblique muscle on paretic side & 17 & & \\
\hline Underaction of inferior rectus muscle on paretic side & 9 & & \\
\hline Overaction of superior oblique muscle on normal side & 2 & & \\
\hline Underaction of inferior oblique muscle on normal side & 1 & & \\
\hline Underaction of superior rectus muscle on normal side & 2 & & \\
\hline No abnormal eye movement & 4 & & \\
\hline
\end{tabular}




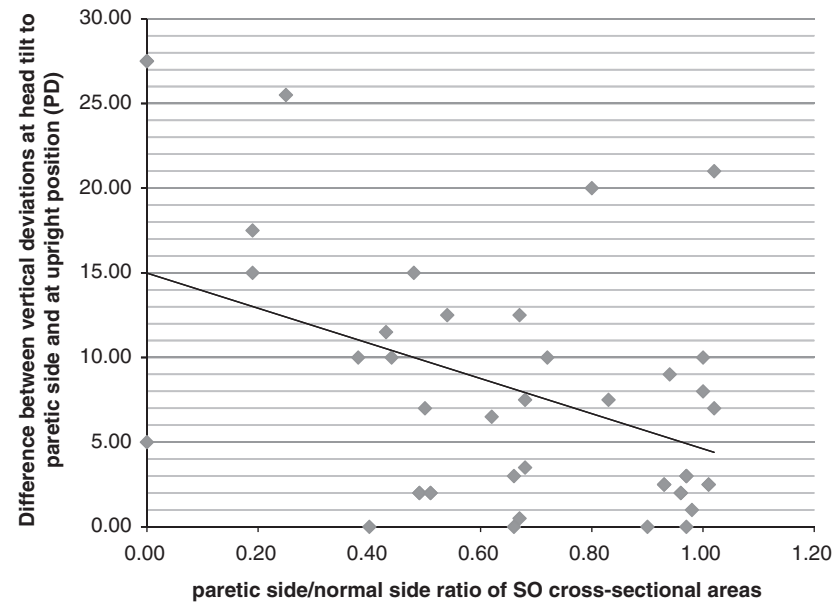

Figure 2 Significant inverse correlation of paretic side/normal side ratios of cross-sectional areas of the superior oblique muscle (SO) with the difference between vertical deviations (PD, prism diopter) at head tilt to the paretic side and at upright position of head $(r=-0.43, P=0.0084, n=37$, Pearson's correlation test). The increase in vertical deviations, elicited by head tilt to the paretic side, is greater when paretic side/normal side ratios of crosssectional areas of the superior oblique muscle are smaller, namely, the superior oblique muscles are more hypoplastic. A full color version of this figure is available at the Journal of Human Genetics journal online.

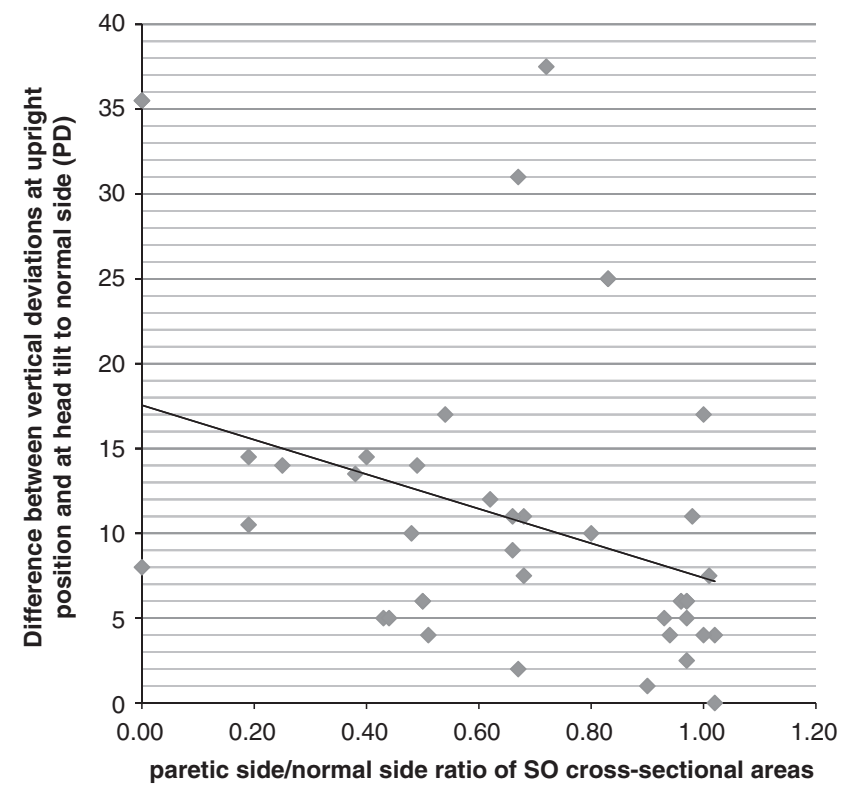

Figure 3 Significant inverse correlation of paretic side/normal side ratios of cross-sectional areas of the superior oblique muscle (SO) with the difference between vertical deviations (PD, prism diopter) at upright head position and at head tilt to the normal side ( $r=-0.34, P=0.038, n=37$, Pearson's correlation test). The decrease in vertical deviations, elicited by head tilt to the normal side, is greater when paretic side/normal side ratios of crosssectional areas of the superior oblique muscle are smaller, namely, the superior oblique muscles are more hypoplastic. A full color version of this figure is available at the Journal of Human Genetics journal online.

excyclodeviation on both the sides $(P=0.018)$ compared with 23 patients with no polymorphism.

In the analysis, regarding eye movement abnormalities (Table 1), 35 patients were divided into two groups based on the presence (27

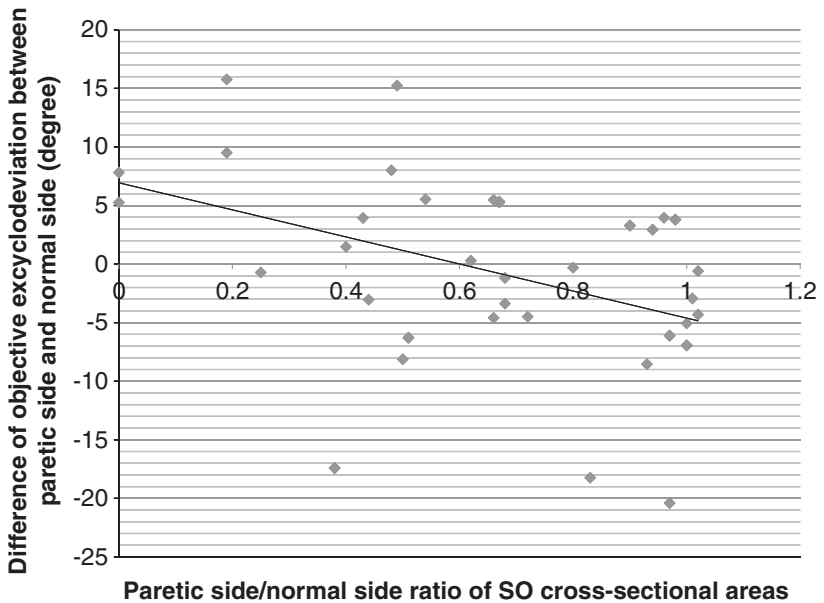

Figure 4 Significant inverse correlation of paretic side/normal side ratios of cross-sectional areas of the superior oblique muscle (SO) with the difference of angles of objective excyclodeviation between the paretic side and normal side (paretic-side value minus normal-side value, $r=-0.43, P=0.009$, $n=37$, Pearson's correlation test). Excyclodeviation on the paretic side, relative to excyclodeviation on the normal side, is greater when paretic side/ normal side ratios of cross-sectional areas of the superior oblique muscle are smaller, namely, the superior oblique muscles are more hypoplastic. A full color version of this figure is available at the Journal of Human Genetics journal online.

Table 2 ARIX and/or PHOX2B gene polymorphisms in 35 patients with idiopathic superior oblique muscle palsy with the genotypes obtained

\begin{tabular}{lcc}
\hline & \multicolumn{2}{c}{ Number of patients } \\
\cline { 2 - 3 } $\begin{array}{l}\text { Polymorphisms in the numbering } \\
\text { of mRNA sequence }\end{array}$ & $\begin{array}{c}\text { With } \\
\text { polymorphism }\end{array}$ & $\begin{array}{c}\text { Without } \\
\text { polymorphism }\end{array}$ \\
\hline ARIX 153G to A in 5'-UTR of exon 1 & 5 & 30 \\
(With PHOX2B 1121A to C) & 2 & \\
PHOX2B polymorphisms in total & 12 & 23 \\
PHOX2B 1121A to C in exon 3 & 10 & 25 \\
(With ARIX 153G to A) & 2 & \\
PHOX2B 1106A to C in exon 3 & 1 & 34 \\
PHOX2B 15bp deletion (1124-1138) in exon 3 & 1 & 34 \\
\hline
\end{tabular}

5'-UTR, 5'-untranslated region of mRNA.

patients) or the absence ( 8 patients) of underaction of the superior oblique muscle or overaction of the inferior oblique muscle or their combinations on the paretic side. These two groups of the patients did not have significant difference in the presence of $A R I X$ or PHOX $2 B$ polymorphisms or their combinations.

\section{DISCUSSION}

The goals of this study are twofold: (1) to understand the relationship among quantitative phenotypes of idiopathic superior oblique muscle palsy, and (2) to search for the role of ARIX and PHOX2B gene polymorphisms in these quantitative phenotypes by analyzing the genotype-phenotype correlation of the disease. The diagnostic term 'congenital superior oblique muscle palsy' is used for younger patients who manifest head tilt from earlier years of the age. The muscle palsy is sometimes diagnosed in adult patients who have been maintaining binocular fusion by head tilt to the contralateral side and also by a 


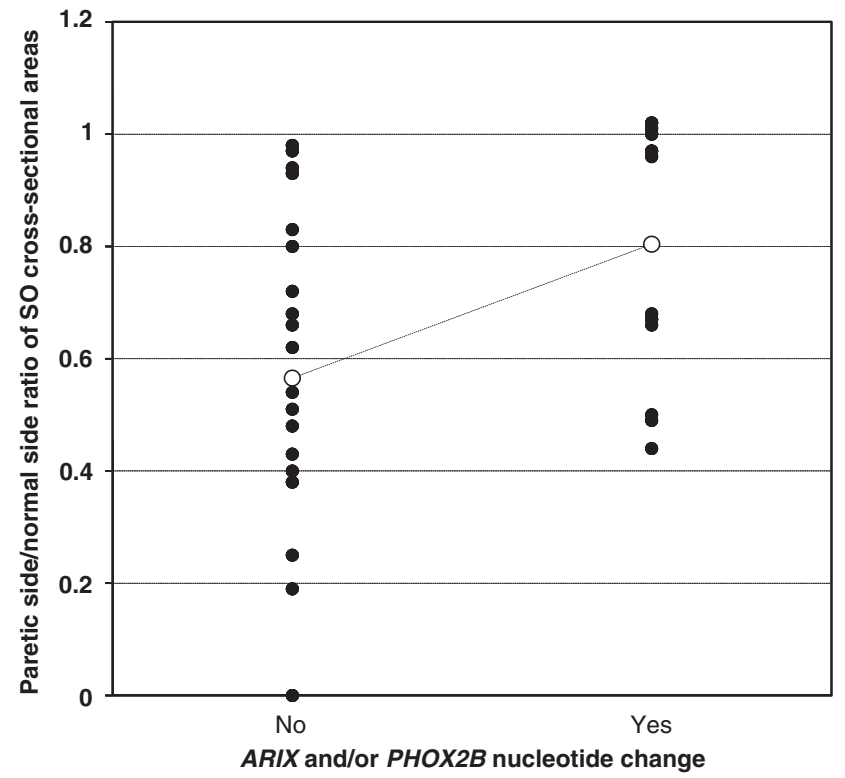

Figure 5 The presence or the absence of $A R I X$ and/or $P H O X 2 B$ gene polymorphisms versus paretic side/normal side ratios of cross-sectional areas of the superior oblique muscle. Of 35 patients with idiopathic superior oblique muscle palsy with the genotypes obtained, 15 patients with $A R I X$ and/or $P H O X 2 B$ polymorphisms have significantly larger paretic side/normal side ratios of cross-sectional areas of the superior oblique muscle (SO) compared with 20 patients with no polymorphism. Namely, the patients with the gene polymorphisms have less hypoplastic muscles compared with those with no polymorphisms ( $P=0.017$, Mann-Whitney $U$-test). White circles represent means.

large amplitude of vertical fusion. The term 'idiopathic superior oblique muscle palsy' is used in this study to include both the groups of patients, in place of the term 'congenital superior oblique muscle palsy'.

Magnetic resonance imaging has served as a diagnostic aid to reveal structural abnormalities of the superior oblique muscle such as the hypoplasia and aplasia, ${ }^{5-14}$ but varying degrees of the muscle hypoplasia present a diagnostic puzzle for its interpretation as a phenotype. ${ }^{14}$ We previously demonstrated that paretic-side/normal-side ratios of cross-sectional areas of the superior oblique muscle in usual coronal sections of magnetic resonance imaging with both the eyes closed gave a good estimation of the muscle hypoplasia, ${ }^{14}$ as did the standardized method, using quasi-coronal sections perpendicular to the long axis of the orbit, obtained with one eye open and fixating at a visual target. ${ }^{24}$ We used the paretic-side/normal-side ratios of cross-sectional areas of the muscle as a quantitative phenotype in this study.

The idiopathic superior oblique muscle palsy is an established clinical entity and its diagnosis is based primarily on eye movement abnormalities, such as underaction of the superior oblique muscle, overaction of the inferior oblique muscle and the Bielschowsky head tilt phenomenon. The Bielschowsky head tilt phenomenon indicates that the extent of hypertropia increases when the patient's head is tilted to the paretic side of the superior oblique muscle compared with hypertropia at upright position of the head. However, this phenomenon is not necessarily a unique feature of the idiopathic superior oblique muscle palsy. ${ }^{25}$ In this study, we chose, as quantitative phenotypes, the differences between vertical deviations at upright position and at head tilt to the paretic side or the normal side.
Patients with idiopathic superior oblique muscle palsy do not notice cyclotropia such as tilting of the image viewed in one eye, compared with the other eye, probably due to a large amplitude of cyclovertical fusion. This is the reason why we used objective cyclodeviations on the paretic side, but not, subjective cyclodeviations, as a phenotype. We chose in this study, as quantitative phenotypes, the sum of angles of excyclodeviations on both the sides and the difference between angles of excyclodeviations on the paretic side and on the normal side in addition to actual angles of objective excyclodeviation on the paretic side. We used the paretic-side minus normal-side values for the difference in angles of excyclodeviations, irrespective of the dominant (fixating) eye either on the paretic side or on the normal side. This is because the angle of objective excyclodeviation in each eye was determined in the monocularly viewing condition with a fixation target.

In the present study, to analyze the correlation among the quantitative phenotypes in idiopathic superior oblique muscle palsy, we, for the first time, found that the more hypoplastic was the superior oblique muscle, the greater were both the increase in vertical deviations elicited by the head tilt to the paretic side and the decrease in vertical deviations elicited by the head tilt to the normal side compared with upright position of the head. In contrast, vertical deviations at upright position, by themselves, did not show the correlation with the extent of the muscle hypoplasia. These facts suggest that the Bielschowsky head tilt phenomenon might be influenced by the extent of the superior oblique muscle hypoplasia and that the changes in vertical deviations by head tilt to the paretic side or to the normal side would be used as quantitative phenotypes in idiopathic superior oblique muscle palsy. In previous studies, the difference between vertical deviations at head tilt to the paretic side and at upright head position was suggested as a better indicator of the superior oblique muscle function in patients with idiopathic superior oblique muscle palsy. ${ }^{26-28}$ The suggestion was proven true by the present results as mentioned above.

In the other previous study, excyclodeviation was measured to show no change at head tilt in patients with superior oblique muscle palsy. ${ }^{29}$ In contrast, this study confirmed, for the first time, that the difference of angles of excyclodeviation between the paretic side and normal side, but not actual angles of excyclodeviation on the paretic side, showed the relationship with the extent of the muscle hypoplasia. As the fovea is located inferior to the optic disc center in the normal condition, interpersonal fluctuations in excyclodeviation might be erased by reducing the angle on the normal side from the angle on the paretic side. Therefore, the difference of angles of excyclodeviation between the paretic side and normal side could be used as a more reliable indicator for the quantitative phenotype than actual angles of excyclodeviation on the paretic side, in idiopathic superior oblique muscle palsy.

In the plan of this study to analyze the genotype-phenotype correlation in idiopathic superior oblique muscle palsy, we hypothesized that ARIX and PHOX2B gene polymorphisms might be related to superior oblique muscle hypoplasia. To this end, we used the paretic-side/normal-side ratios of cross-sectional areas of the superior oblique muscle as a quantitative phenotype to show a continuous spectrum of the muscle hypoplasia. ${ }^{14}$ Contrary to the hypothesis, the present study revealed that the patients with polymorphisms had less hypoplastic muscles compared with the patients with no polymorphisms. The patients with ARIX polymorphisms only also showed a tendency to have less hypoplastic muscles. These results suggest that the ARIX and $P H O X 2 B$ polymorphisms might have an additive effect on the development of less hypoplastic muscles as a 
Table 3 Genotype-phenotype correlation in 35 patients with idiopathic superior oblique muscle palsy

\begin{tabular}{|c|c|c|c|c|c|c|}
\hline Phenotype & Gene & Polymorphism & $\begin{array}{l}\text { Number of } \\
\text { patients }\end{array}$ & Mean \pm s.d. & $\begin{array}{l}\text { Range } \\
\text { (median) }\end{array}$ & $\begin{array}{c}\mathrm{P}- \\
\text { value }\end{array}$ \\
\hline \multirow[t]{2}{*}{ Vertical deviation at upright position (prism diopter) } & \multirow[t]{2}{*}{ ARIX } & Yes & 5 & $18.7 \pm 10.5$ & $4.0-30.0(18.0)$ & \multirow[t]{2}{*}{0.54} \\
\hline & & No & 30 & $21.4 \pm 8.3$ & $10.0-37.5(18.0)$ & \\
\hline \multirow{2}{*}{$\begin{array}{l}\text { Difference between vertical deviations at head tilt to paretic side and at } \\
\text { upright position (prism diopter) }\end{array}$} & \multirow[t]{2}{*}{$A R I X$} & Yes & 5 & $7.7 \pm 8.0$ & $0-21.0(7.0)$ & \multirow[t]{2}{*}{0.55} \\
\hline & & No & 30 & $8.2 \pm 7.1$ & $0-27.5(7.3)$ & \\
\hline \multirow{2}{*}{$\begin{array}{l}\text { Difference between vertical deviations at upright position and at head tilt } \\
\text { to normal side (prism diopter) }\end{array}$} & \multirow[t]{2}{*}{$A R I X$} & Yes & 5 & $4.6 \pm 3.7$ & $0-9.0(4.0)$ & \multirow[t]{2}{*}{0.034} \\
\hline & & No & 30 & $12.2 \pm 9.2$ & $2-37.5(10.5)$ & \\
\hline \multirow[t]{2}{*}{ Angle of objective excyclodeviation on paretic side (degree) } & \multirow[t]{2}{*}{$A R I X$} & Yes & 5 & $6.4 \pm 6.9$ & -5.2 to $12.3(8.4)$ & \multirow[t]{2}{*}{0.38} \\
\hline & & No & 30 & $10.2 \pm 5.0$ & -3.2 to $19.0(9.5)$ & \\
\hline \multirow[t]{2}{*}{ Sum of angles of objective excyclodeviation on both the sides (degree) } & \multirow[t]{2}{*}{$A R I X$} & Yes & 5 & $19.2 \pm 6.9$ & $9.9-28.6(19.3)$ & \multirow[t]{2}{*}{0.51} \\
\hline & & No & 30 & $20.5 \pm 6.0$ & $8.9-30.8(21.9)$ & \\
\hline \multirow{2}{*}{$\begin{array}{l}\text { Difference of objective excyclodeviation between paretic side and normal } \\
\text { side (degree) }\end{array}$} & \multirow[t]{2}{*}{$A R I X$} & Yes & 5 & $-6.2 \pm 8.1$ & -20.4 to $0.59(-4.3)$ & \multirow[t]{2}{*}{0.18} \\
\hline & & No & 30 & $-0.25 \pm 7.9$ & -18.2 to $15.8(-0.01)$ & \\
\hline \multirow{2}{*}{$\begin{array}{l}\text { Paretic-side/normal-side ratio of cross-sectional areas of superior oblique } \\
\text { muscle }\end{array}$} & \multirow[t]{2}{*}{$A R I X$} & Yes & 5 & $0.87 \pm 0.18$ & $0.66-1.02(0.97)$ & \multirow[t]{2}{*}{0.056} \\
\hline & & No & 30 & $0.63 \pm 0.30$ & $0.00-1.01(0.67)$ & \\
\hline \multirow[t]{2}{*}{ Vertical deviation at upright position (prism diopter) } & \multirow[t]{2}{*}{$\mathrm{PHOX} 2 \mathrm{~B}$} & Yes & 12 & $20.5 \pm 8.1$ & $10.0-35.0(17.0)$ & \multirow[t]{2}{*}{0.52} \\
\hline & & No & 23 & $21.2 \pm 8.9$ & $4.0-37.5(19.0)$ & \\
\hline \multirow{2}{*}{$\begin{array}{l}\text { Difference between vertical deviations at head tilt to paretic side and at } \\
\text { upright position (prism diopter) }\end{array}$} & \multirow[t]{2}{*}{$\mathrm{PHOX} 2 \mathrm{~B}$} & Yes & 12 & $5.7 \pm 4.0$ & $0.5-12.5(5.0)$ & \multirow[t]{2}{*}{0.32} \\
\hline & & No & 23 & $9.4 \pm 8.2$ & $0-27.5(7.5)$ & \\
\hline Difference between vertical deviations at upright position and at head tilt & PHOX2B & Yes & 12 & $9.5 \pm 8.0$ & $2.0-31.0(6.8)$ & 0.40 \\
\hline to normal side (prism diopter) & & No & 23 & $12.0 \pm 9.5$ & 0-37.5 (11.0) & \\
\hline Angle of objective excyclodeviation on paretic side (degree) & PHOX2B & Yes & 12 & $11.6 \pm 4.4$ & $5.3-18.1(9.8)$ & 0.20 \\
\hline & & No & 23 & $8.7 \pm 5.6$ & -5.3 to $19.0(8.4)$ & \\
\hline Sum of angles of objective excyclodeviation on both the sides (degree) & PHOX2B & Yes & 12 & $24.0 \pm 5.1$ & $13.4-30.8(23.6)$ & 0.018 \\
\hline & & No & 23 & $18.4 \pm 5.7$ & 8.9-28.3 (18.1) & \\
\hline Difference of objective excyclodeviation between paretic side and normal & PHOX2B & Yes & 12 & $-0.68 \pm 6.8$ & -8.1 to $15.2(-3.0)$ & 0.52 \\
\hline side (degree) & & No & 23 & $-1.3 \pm 8.8$ & -20.4 to $15.8(-0.3)$ & \\
\hline Paretic side/normal side ratio of cross-sectional areas of superior oblique & PHOX2B & Yes & 12 & $0.75 \pm 0.22$ & $0.44-1.01(0.68)$ & 0.26 \\
\hline muscle & & No & 23 & $0.62 \pm 0.32$ & $0-1.02(0.66)$ & \\
\hline Vertical deviation at upright position (prism diopter) & $A R I X$ & Yes & 15 & $19.7 \pm 8.6$ & $4.0-35.0(17.0)$ & 0.49 \\
\hline & $\begin{array}{l}\text { and/or } \\
\text { PHOX2B }\end{array}$ & No & 20 & $21.9 \pm 8.6$ & $10.0-37.5(19.0)$ & \\
\hline Difference between vertical deviations at head tilt to paretic side and at & $A R I X$ & Yes & 15 & $6.4 \pm 5.6$ & $0-21.0(7.0)$ & 0.32 \\
\hline upright position (prism diopter) & $\begin{array}{l}\text { and/or } \\
P H O X 2 B\end{array}$ & No & 20 & $9.4 \pm 8.1$ & $0-27.5(8.3)$ & \\
\hline Difference between vertical deviations at upright position and at head tilt & $A R I X$ & Yes & 15 & $8.0 \pm 7.7$ & $0-31.0(6.0)$ & 0.028 \\
\hline to normal side (prism diopter) & $\begin{array}{l}\text { and/or } \\
\text { PHOX2B }\end{array}$ & No & 20 & $13.4 \pm 9.4$ & $4.0-37.5(11.0)$ & \\
\hline Angle of objective excyclodeviation on paretic side (degree) & $A R I X$ & Yes & 15 & $10.0 \pm 5.9$ & -5.3 to $18.1(9.6)$ & 0.52 \\
\hline & $\begin{array}{l}\text { and/or } \\
\text { PHOX2B }\end{array}$ & No & 20 & $9.4 \pm 5.0$ & -3.2 to $19.0(8.5)$ & \\
\hline
\end{tabular}


Table 3 (Continued)

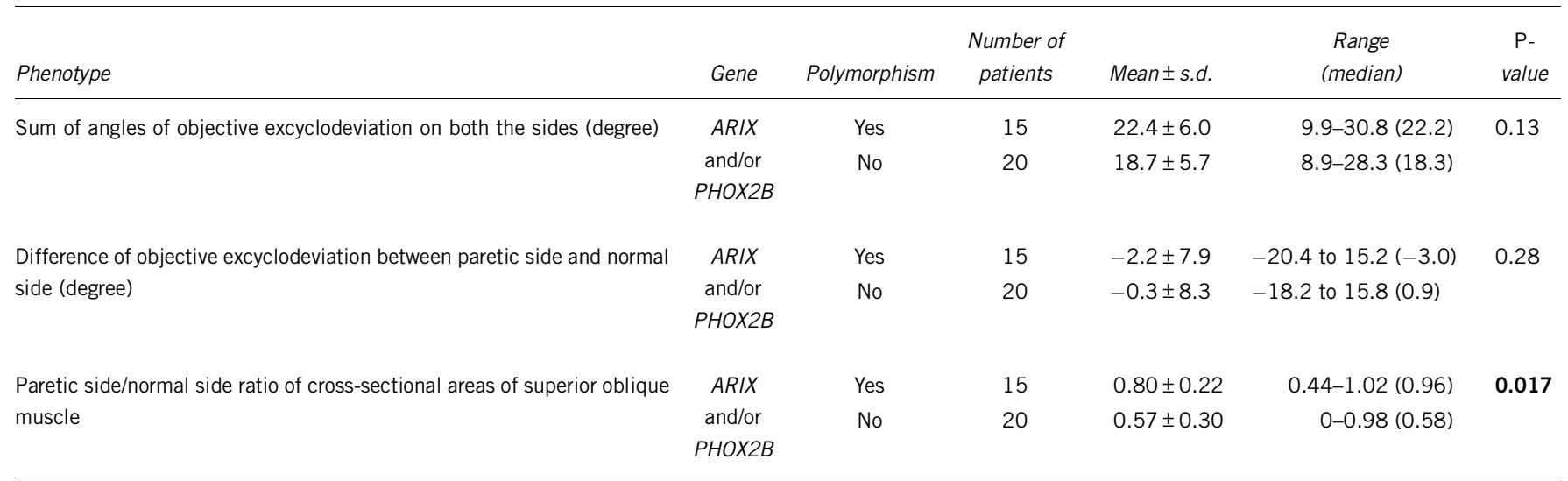

$P$-values in Mann-Whitney $U$-test. Significant $P$-values $(P<0.05)$ are highlighted by bold font.

quantitative phenotype. The $A R I X$ and $P H O X 2 B$ genes would have a certain role in the muscle development, but the other unknown gene might be also involved in severer hypoplasia of the superior oblique muscle.

Previous researchers have suggested that idiopathic superior oblique muscle palsy with the muscle hypoplasia is designated as true muscle palsy while the palsy without the muscle hypoplasia is designated as simulated muscle palsy. ${ }^{30}$ Abnormalities in the connective tissues, supporting the extraocular muscles in the orbit, called 'muscle pulley' have been suggested as a possible cause for this simulated muscle palsy. A major problem in this classification is the unnatural divide into hypoplastic muscles and non-hypoplastic muscles under the circumstances that the superior oblique muscles actually show the continuous spectrum of hypoplasia from aplasia to the near-normal muscle. ${ }^{14}$ The situation provides one example whether to interpret a certain clinical trait either as a quantitative phenotype or as the presence or the absence of a phenotype. Based on the previous author's definition of the term, ${ }^{30}$ the ARIX and $P H O X 2 B$ polymorphisms might be related with the development of simulated superior oblique muscle palsy that is associated with less hypoplastic muscles. In other words, the ARIX and $P H O X 2 B$ polymorphisms might have a role in the abnormal development of connective tissues that support the extraocular muscles.

It should be noted that only the presence of the $P H O X 2 B$ polymorphisms, but not the presence of the ARIX polymorphisms, was related with larger sum of angles of excyclodeviation on both sides. This genotype-phenotype correlation would be in contradiction to the phenotypic relation of more hypoplastic muscles with larger angles of excyclodeviation on the paretic side relative to the normal side. The function of the superior oblique muscle, manifesting as vertical deviations and cyclodeviations, is not necessarily related directly to the structure of the muscle such as the hypoplasia. ${ }^{31}$ The discrepancy between the structure and function in this study would be understandable from this point of view. Furthermore, both ARIX and $P H O X 2 B$ genes are expressed in trochlear and oculomotor nuclei of the brainstem, and their gene products interact with each other. ${ }^{19,20}$ The balance between the two genes might be changed by the polymorphisms and, hence, might influence the phenotypes in a different way.

In conclusion, this study, for the first time, identified reliable quantitative phenotypes by phenotype-phenotype correlation analysis, and also revealed the role of the ARIX and PHOX2B polymorphisms in the muscle hypoplasia by genotype-phenotype correlation analysis in idiopathic superior oblique muscle palsy. From the viewpoint of the correlation among quantitative phenotypes in idiopathic superior oblique muscle palsy, the larger the increase of vertical deviation elicited by head tilt to the paretic side, the larger was the decrease of vertical deviation elicited by head tilt to the normal side, and the greater was the angle of objective excyclodeviation on the paretic side relative to the normal side, more hypoplastic was the superior oblique muscle. From the viewpoint of the genotype-phenotype correlation, the patients with ARIX and/or $P H O X 2 B$ polymorphisms had less hypoplastic superior oblique muscles as a quantitative phenotype. A major limitation of this study is selection bias of patients as the study involved only the patients both with complete sets of clinical data and with their genomic DNA obtained after their consent to the study. Indeed, three patients with polymorphisms in the promoter region of the ARIX gene, as we reported previously, ${ }^{18,19}$ were not included in this study because of the lack of their clinical data. These inadvertent exclusions of patients would be a potential weak point in analyzing the genotype-phenotype correlation in this study. In the future, the quantitative phenotypes, identified in this study, would be used for quantitative trait locus analysis for idiopathic superior oblique muscle palsy.

\section{ACKNOWLEDGEMENTS}

This study was supported in part by a grant-in-aid for scientific research from the Ministry of Education, Science, Sports, Culture, and Technology of Japan (No. 22591964).

1 Bhola, R. M., Horne, G. V., Squirrell, D. M., Chan, T. K. \& Kumar, D. Autosomal dominant congenital superior oblique palsy. Eye 15, 479-484 (2001).

2 Botelho, P. J. \& Giangiacomo, J. G. Autosomal-dominant inheritance of congenital superior oblique palsy. Ophthalmology 103, 1508-1511 (1996).

3 Harris Jr, D. J., Memmen, J. E., Katz, N. N. \& Parks, M. M. Familial congenital superior oblique palsy. Ophthalmology 93, 88-90 (1986).

4 Astle, W. F. \& Rosenbaum, A. L. Familial congenital fourth cranial nerve palsy. Arch. Ophthalmol. 103, 532-535 (1985).

5 Imai, S., Matsuo, T., Itoshima, E. \& Ohtsuki, H. Clinical features, ARIX and PHOX2B nucleotide changes in three families with congenital superior oblique muscle palsy. Acta Med. Okayama 62, 45-53 (2008).

6 Sawa, M. Absence of the right superior oblique muscle: a case report. Jpn. J. Ophthalmol. 22, 178-183 (1978).

7 Matsuo, T., Ohtsuki, H., Sogabe, Y., Konishi, H., Takenawa, K. \& Watanabe, Y. Vertical abnormal retinal correspondence in three patients with congenital absence of the superior oblique muscle. Am. J. Ophthalmol. 106, 341-345 (1988). 
8 Helveston, E. M., Krach, D., Plager, D. A. \& Ellis, F. D. A new classification of superior oblique palsy based on congenital variations in the tendon. Ophthalmology 99, 1609-1615 (1992).

9 Plager, D. A. Tendon laxity in superior oblique palsy. Ophthalmology 99, 1032-1038 (1992).

10 Demer, J. L. \& Miller, J. M. Magnetic resonance imaging of the functional anatomy of the superior oblique muscle. Invest. Ophthalmol. Vis. Sci. 36, 906-913 (1995).

11 Sato, M. Magnetic resonance imaging and tendon anomaly associated with congenital superior oblique palsy. Am. J. Ophthalmol. 127, 379-387 (1999).

12 Chan, T. K. \& Demer, J. L. Clinical features of congenital absence of the superior oblique muscle as demonstrated by orbital imaging. J. AAPOS 3, 143-150 (1999).

13 Sato, M., Iwata, E. A., Takai, Y., Hikoya, A. \& Koide, Y. M. Superior oblique palsy with class III tendon anomaly. Am. J. Ophthalmol. 146, 385-394 (2008).

14 Uchiyama, E., Matsuo, T., Imai, S. \& Itoshima, E. Paretic side/normal side ratios of cross-sectional areas of the superior oblique muscle vary largely in idiopathic superior oblique palsy. Am. J. Ophthalmol. 149, 508-512 (2010).

15 Engle, E. C. Genetic basis of congenital strabismus. Arch. Ophthalmol. 125, 189-195 (2007).

16 Yamada, K., Andrews, C., Chan, W. M., McKeown, C. A., Magli, A., De Berardinis, T. et al. Heterozygous mutations of the kinesin KIF21A in congenital fibrosis of the extraocular muscles type1(CFEOM1). Nat. Genet. 35, 318-321 (2003).

17 Nakano, M., Yamada, K., Fain, J., Sener, E. C., Selleck, C. J., Awad, A. H. et al. Homozygous mutations in ARIX(PHOX2A) result in congenital fibrosis of the extraocular muscles type 2. Nat. Genet. 29, 315-320 (2001).

18 Jiang, Y., Matsuo, T., Fujiwara, H., Hasebe, S., Ohtsuki, H. \& Yasuda, T. ARIX gene polymorphisms in patients with congenital superior oblique muscle palsy. Br. J. Ophthalmol. 88, 263-267 (2004).

19 Jiang, Y., Matsuo, T., Fujiwara, H., Hasebe, S., Ohtsuki, H. \& Yasuda, T. ARIX and PHOX2B polymorphisms in patients with congenital superior oblique muscle palsy. Acta Med. Okayama 59, 55-62 (2005).
20 Pattyn, A., Morin, X., Cremer, H., Goridis, C. \& Brunet, J F. Expression and interactions of the two closely related homeobox genes Phox2a and Phox $2 b$ during neurogenesis. Development 124, 4065-4075 (1997).

21 Horikawa, A., Hirai, Y., Kono, R., Hasebe, S. \& Ohtsuki, H. Evaluation of cyclodeviation by scanning laser ophthalmoscope. Comparison of subjective and objective cyclodeviation in vertical strabismus. (in Japanese). Rinsho Ganka (Jpn. J. Clin. Ophthalmol.) 54, 85-88 (2000).

22 Horikawa, A., Hirai, Y., Kono, R., Hasebe, S. \& Ohtsuki, H. Sensory adaptation to cyclodeviations in superior oblique palsy. (in Japanese). Rinsho Ganka (Jpn. J. Clin. Ophthalmol.) 54, 1447-1450 (2000).

23 Bixenman, W. W. \& von Noorden, G. K. Apparent foveal displacement in normal subjects and in cyclotropia. Ophthalmology 89, 58-62 (1982).

24 Demer, J. L., Miller, J. M., Koo, E. Y. \& Rosenbaum, A. L. Quantitative magnetic resonance morphometry of extraocular muscles: a new diagnostic tool in paralytic strabismus. J. Pediatr. Ophthalmol. Strabismus 31, 177-188 (1994).

25 Kushner, B. J. Errors in the three-step test in the diagnosis of vertical strabismus. Ophthalmology 96, 127-132 (1989).

26 Hamasaki, I., Hasebe, S., Furuse, T. \& Ohtsuki, H. Relationship between static ocular counterroll and Bielschowsky head tilt phenomenon. Invest. Ophthalmol. Vis. Sci. 51, 201-206 (2010).

27 Kono, R., Okanobu, H., Ohtsuki, H. \& Demer, J. L. Absence of relationship between oblique muscle size and Bielschowsky head tilt phenomenon in clinically diagnosed superior oblique palsy. Invest. Ophthalmol. Vis. Sci. 50, 175-179 (2009).

28 Ohtsuki, H., Hasebe, S., Kono, R., Yamane, T., Fujiwara, H. \& Shiraga, F. Large Bielschowsky head-tilt phenomenon and inconspicuous vertical deviation in the diagnostic positions in congenital superior oblique palsy. Am. J. Ophthalmol. 130, 854-856 (2000).

29 Kushner, B. J. The influence of head tilt on ocular torsion in patients with superior oblique muscle palsy. . J. AAPOS 13, 132-135 (2009).

30 Kono, R., Okanobu, H., Ohtsuki, H. \& Demer, J. L. Displacement of the rectus muscle pulleys simulating superior oblique palsy. . Jpn. J. Ophthalmol. 52, 36-43 (2008).

31 Kushner, B. J. Incomitant strabismus. Does extraocular muscle form denote function? Arch. Ophthalmol. 128, 1604-1609 (2010). 